\title{
EDUCATION AND TRAINING Integrating sustainability into postgraduate medical education
}

\author{
Authors: Vanita Gandhi, ${ }^{\mathrm{A}}$ Nada Al-Hadithy, ${ }^{\mathrm{B}}$ Anya Göpfert, ${ }^{\mathrm{C}}$ Katie Knight, ${ }^{\mathrm{D}}$ Maria van Hove ${ }^{\mathrm{E}}$ and Peter Hockey
}

The delivery of healthcare is a major contributor to the climate crisis, with the NHS being the largest public sector contributor of carbon emissions in the UK. Physicians have an important role to play in the fight against climate change through the practice of sustainable healthcare. This involves maintaining the current and future quality of healthcare through balancing environmental, social and financial constraints. To this end, integrating these skills into medical education is crucial. A large number of medical schools have already embedded planetary health and sustainability theory into their curriculum, however, there is no formal sustainability curriculum in postgraduate education and training. This is vital for enabling clinicians to translate sustainability theory taught at undergraduate level into clinical practice. This article proposes which topics should be included in a postgraduate sustainability curriculum and explores various methods that could be used to incorporate these into the current educational framework.

KEYWORDS: Sustainability, sustainable healthcare, climate change, curriculum, postgraduate medical education

DOI: $10.7861 /$ fhj.2020-0042

\section{Introduction}

Climate change is the biggest global health threat of the 21st century. The health consequences are becoming progressively more visible: extreme weather events such as flooding leaves millions of people without clean water and food; more frequent heat waves and wildfires cause cardiovascular and respiratory symptoms; rapid spread of infectious diseases; and increasing air pollution, which is strongly linked to heart disease, asthma, stroke and lung cancer. ${ }^{1-3}$

Authors: Anational medical director's clinical fellow and clinical oncology registrar, Health Education England, London, UK; ${ }^{B}$ plastic surgery registrar, Southmead Hospital, Bristol, UK; ${ }^{C}$ public health registrar, Oxford University Hospitals NHS Foundation Trust, Oxford, UK; ${ }^{D}$ paediatric emergency medicine consultant, North Middlesex

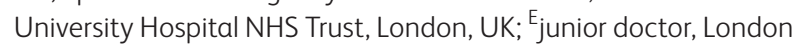
School of Hygiene and Tropical Medicine, London, UK; ${ }^{\text {p }}$ professor of clinical education, University of Sydney, Sydney, Australia
Although the mission of the health sector is to protect and promote health, the delivery of healthcare remains a significant contributor to the climate crisis. The NHS is the largest public sector contributor of carbon emissions. It accounts for $5 \%$ of all traffic on UK roads, $5 \%$ of England's national carbon footprint and $4 \%$ of the country's solid waste. ${ }^{4,5}$ Great efforts have been made to 'greening' the health sector with hospital estates paying particular attention to improving waste facilities, water and energy systems, transport options, and adapting building infrastructure. However, only a third of emissions come from direct energy use from buildings and staff/patient travel. The remaining two-thirds of emissions are attributable to decisions made in clinical care, particularly related to procurement of goods and services. ${ }^{6}$

As physicians, our professional duty is to help maintain the health and wellbeing of the populations we serve. Delivering sustainable healthcare means maintaining both the current and future quality of healthcare through balancing social, environmental and financial constraints. ${ }^{7}$ As a trusted profession that oversees a patient's journey through the health system, doctors are uniquely placed to help redesign healthcare to benefit the patient, planet and NHS 'purse'. Sustainable healthcare education is emerging as a new and necessary response to this need. The General Medical Council (GMC) requires that newly qualified doctors are able to apply the principles, methods and knowledge of sustainable healthcare to clinical practice. ${ }^{8}$ There are currently 33 medical schools recognised by the GMC, and despite most having very diverse curricula structures, a large number have already embedded planetary health and sustainable healthcare skills into their curricula in the form of lectures and student-selected component modules. ${ }^{9}$ In order to effectively implement the GMC's recommendations, not only should sustainability be taught to medical students, but it also must be incorporated into education for postgraduate trainee doctors.

Postgraduate medical education typically builds on material taught in medical school, translating theory into clinical practice; sustainability education is no different. The aim of a postgraduate sustainability curriculum should be to create a generation of practitioners who automatically consider the social, environmental and economic impacts of their clinical decisions and service development. This article considers the approach to postgraduate healthcare sustainability education in two parts: how doctors can practise sustainable healthcare and how this knowledge is best delivered. 


\section{Principles}

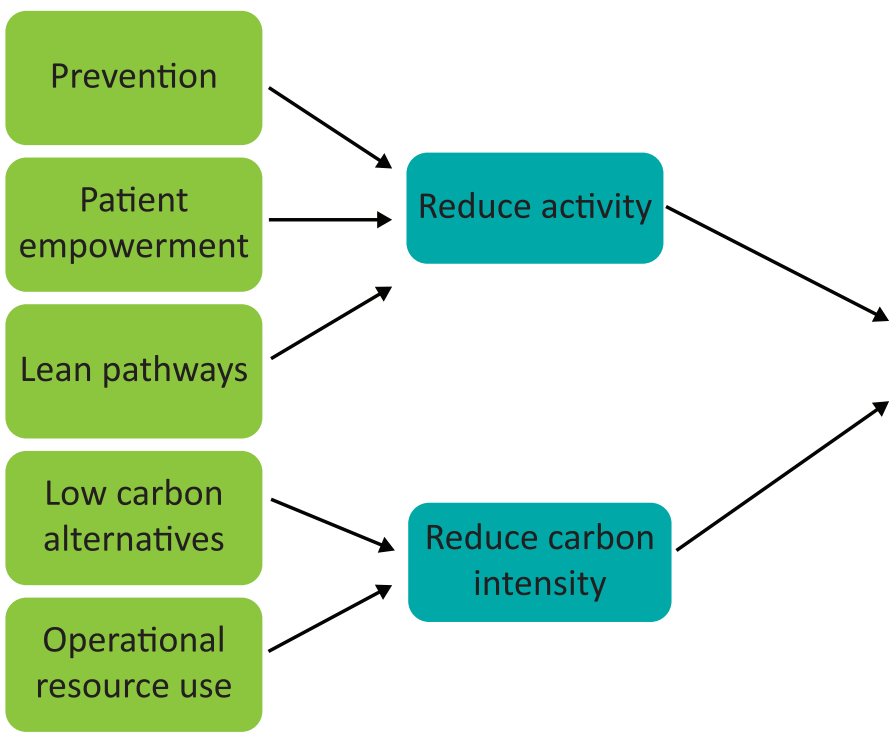

Outcome

\section{Reduce carbon without reducing health}

\section{How do we practise sustainable healthcare?}

There are a number of specific interventions that have environmental benefits across healthcare, for example switching patients from metered dose inhalers to dry powder inhalers. These are important changes that have a significant impact on reducing carbon emissions, however all clinical work is best considered for its impact on sustainability at a patient, service and/or system level. A broader framework for practising sustainable healthcare can be applied across all specialties by using the principles for sustainable clinical practice published by the Centre for Sustainable Healthcare (Fig 1). ${ }^{10}$ As well as switching to low carbon alternatives, the four other domains are prevention; patient empowerment and selfcare; streamlining care and efficiency of clinical pathways; and improving use of operational resources. ${ }^{11}$

Fig 1 demonstrates how the five principles of sustainable healthcare directly contribute to delivery of sustainable clinical practice through reducing wasteful healthcare activity and demand, and reducing the environmental impact of the remaining healthcare activities. This overall reduces the carbon impact of clinical practice and health services while maintaining high standards of care.

\section{Prevention}

Promotion of public health and preventative medicine reduces the overall burden of disease, and therefore reduces demands on the healthcare system, such as frequency of hospital admissions and appointments.

> Example: Smoking cessation initiatives to reduce burden of vascular disease and cancer.

\section{Patient empowerment and self-care}

Educating and encouraging patients to take a bigger role in managing their health, especially those with chronic disease, will also reduce demand on the healthcare system.
Fig 1. Modified driver diagram of the Centre for Sustainable Healthcare principles of sustainable clinical practice. Adapted with permission from Mortimer F. The sustainable physician. Clin Med 2010;10:110-11.
> Example: Teaching patients with heart failure to weigh themselves and change diuretic doses to avoid hospital admissions with heart failure / dehydration.

\section{Lean pathways}

Streamlining care and improving efficiency of clinical pathways.

> Example: The COVID-19 response has led to a significant change in practice through increasing use of telecommunications rather than conventional outpatient clinics, which reduces staff requirement, patient travel and waiting times. Other positive changes to healthcare delivery which have arisen from this pandemic should be explored.

\section{Low carbon alternatives}

Switch to using treatment and technologies with lower environmental impact.

> Example: Increasing use of regional and total intravenous anaesthesia and, for where inhaled anaesthetics are required, minimising the carbon footprint by avoiding use of desflurane and nitrous oxide, which are potent greenhouse gases.

\section{Operational resource use}

Improving use of resources by minimising waste (including medications), energy and travel.

> Example: Minimising waste through reducing packaging or water consumption for a given procedure, improving energy efficiency of buildings and using renewable energy.

\section{How should we incorporate sustainability into postgraduate medical education?}

Postgraduate medical education in the UK follows a typical framework for most specialties. One option for introducing 
sustainability education would be to incorporate it into existing training models, for example, using a section of the electronic portfolio, a method previously used to formally incorporate leadership and patient safety education into postgraduate training. Curriculum items could be developed with support from expert bodies such as the Centre for Sustainable Healthcare, with teaching delivered in the form of modules and courses. Online short courses on climate change and sustainable healthcare could be very attractive to a workforce who have now had their awareness raised about the impact of global disasters, such as the COVID-19 pandemic, and, indeed, such modules are already available in several medical schools across the globe. ${ }^{12}$ Competency on these topics could then be assessed through evidence mapped to defined curricular items. Key underpinning principles of sustainable clinical practice, such as streamlining care and improving clinical pathway efficiency, would be best addressed as part of the UK Foundation Programme curriculum, with specialty-specific principles being tackled during higher training.

However, there are a number of potential challenges. Most trainers are unfamiliar with sustainability in healthcare, having had no education or training in the field themselves. As previously discussed, this needs to be carefully considered to prevent it being perceived as burdensome and result in this important topic being undervalued and considered as merely a tick-box exercise in the portfolio. Such a crucial subject needs to be addressed as a crosscutting theme, which needs to be considered at each stage of postgraduate education, in every subject.

Alternatively, a relatively straightforward way to introduce sustainability into existing educational frameworks could be through quality improvement projects. To avoid increasing the number of assessments required, competence in this topic could be evaluated via the quality improvement assessment tool (QIPAT). Specialties could encourage and guide trainees to design quality improvement projects with outcomes geared towards the five principles of sustainable clinical practice. These projects could be undertaken with the support of individual healthcare trusts' sustainability leads, thus ensuring that the experience is both educational for the trainee and can produce measurable sustainability benefits with meaningful outcomes for the organisation. A section could be added to the existing QIPAT for the trainee to reflect on the environmental, social and financial impacts of their project. This simple intervention would target the vast majority of trainees, for whom participation in high-level quality improvement projects is both mandatory for the annual review of competency progression (ARCP) and valuable for career development.

\section{Conclusion}

By following the five principles of sustainable clinical practice, professionals can strive to improve delivery of sustainable healthcare even where the link to the carbon footprint is less obvious. Education is crucial to this process and, while this topic has recently been introduced into the undergraduate medical curriculum, postgraduate education has not yet caught up. Existing postgraduate educational frameworks such as additional curricular items, assessed via electronic portfolios, are unlikely to be an ideal platform for the incorporation of sustainability teaching due to logistical constraints and trainers' unfamiliarity with the topic. However, by introducing a requirement for reflection on environmental, social and financial endpoints into quality improvement projects, we can encourage trainees to develop the mindset to consider the sustainability impact of their everyday clinical practice - crucial for the next generation of doctors, other healthcare professionals and leaders to tackle the major challenges facing both future health services and the global climate.

\section{References}

1 Costello A, Abbas M, Allen A et al. Managing the health effects of climate change. Lancet 2009;373:1693-733.

2 Watts N, Adger WN, Agnolucci P et al. Health and climate change: policy responses to protect public health. Lancet 2015;386:1861914.

3 Cohen AJ. Air pollution and lung cancer: what more do we need to know? Thorax 2003;58:1010-2.

4 Campbell M, Pierce T. Atmospheric science, anaesthesia, and the environment. BJA Education 2015;15:173-9.

5 Naylor C. What if the NHS were to go carbon-neutral? The King's Fund, 2016. www.kingsfund.org.uk/reports/thenhsif/what-if-carbonneutral-nhs [Accessed 07 April 2020].

6 Hazell B, Robson R. Pharmaceutical waste reduction in the NHS: a best practice compilation paper. London: NHS Business Services Authority; 2015.

7 Mortimer F, Isherwood J, Wilkinson A, Vaux E. Sustainability in quality improvement: redefining value. FHJ 2018;5:88-93.

8 General Medical Council. Outcomes for graduates. GMC, 2018. www.gmc-uk.org/education/standards-guidance-and-curricula/ standards-and-outcomes/outcomes-for-graduates [Accessed 04 April 2020].

9 Tun MS. Fulfilling a new obligation: Teaching and learning of sustainable healthcare in the medical education curriculum. Med Teach 2019:41:1168-77.

10 Mortimer F. The sustainable physician. Clin Med 2010;10:110-11.

11 Mortimer F, Isherwood J, Wilkinson A, Vaux E. Sustainability in quality improvement: redefining value. FHJ 2018;5:88.

12 Madden DL, McLean M, Horton GL. Preparing medical graduates for the health effects of climate change: an Australasian collaboration. Med J Aust 2018;208:291-2.

Address for correspondence: Dr Vanita Gandhi, Health Education England, 4, Stewart House, 32 Russell Square, London WC1B 5DN, UK.

Email:v.gandhi@nhs.net 